\title{
Hot fusion reactions with deformed nuclei for synthesis of superheavy nuclei: an extension of the fusion-by-diffusion model
}

\author{
K. Hagino ${ }^{1,2}$ \\ 1 Department of Physics, Tohoku University, Sendai 980-8578, Japan \\ ${ }^{2}$ Research Center for Electron Photon Science, Tohoku University, 1-2-1 Mikamine, Sendai 982-0826, Japan
}

\begin{abstract}
The fusion-by-diffusion model proposed by Swiatecki et al. [Phys. Rev. C71, 014602 (2005)] has provided a simple and convenient tool to estimate evaporation residue cross sections for superheavy nuclei. I extend this model by taking into account deformation of the target nucleus, and discuss the role of orientation of deformed target in hot fusion reactions at energies around the Coulomb barrier. To this end, I introduce an injection point for the diffusion process over an inner barrier which depends on the orientation angle. I apply this model to the ${ }^{48} \mathrm{Ca}+{ }^{248} \mathrm{Cm}$ reaction and show that the maximum of evaporation residue cross section appears at an energy slightly above the height of the capture barrier for the side collision, for which the effective inner barrier is considerably lower than that for the tip collision, thus enhancing the diffusion probability. I also discuss the energy dependence of the injection point, and show that a large part of the energy dependence found in the previous analyses can be attributed to the deformation effect of a target nucleus.
\end{abstract}

\section{INTRODUCTION}

An investigation of superheavy elements has been one of the most important topics in nuclear physics $1-3]$. It is not only related to a fundamental question: "how heavy element can one define as a nucleus?", but also relevant to many areas of science, including nuclear structure, nuclear reaction, chemistry and nuclear astrophysics [3] . That is, the stability of superheavy elements is intimately related to the shell structure of superheavy nuclei, and an understanding the reaction dynamics is extremely important for a formation of superheavy nuclei. Furthermore, a good understanding of electronic structure as well as chemical properties is necessary to locate superheavy elements at appropriate positions in a periodic table. Fission of heavy and superheavy elements in r-process nucleosynthesis is also an important topic in nuclear astrophysics in order to investigate the origin of elements found in nature [4].

The heaviest element synthesized so far is the element 118 [5], which was recently named oganesson (Og), together with three other superheavy elements, that is, nihonium (Nh: $Z=113$ ) [6, 7], moscovium (Mc: $Z=115$ ) [7], and tennessine (Ts: $Z=117$ ) [8]. These superheavy elements, as well as elements heavier than mendelevium $(Z=101)$, have been synthesized using heavy-ion fusion reactions at energies around the Coulomb barrier [1 3].

It is important to notice here that fusion reactions for superheavy elements are considerably different from fusion in medium-heavy systems [9-12]. Whereas a compound nucleus is formed almost automatically in medium-heavy systems once projectile and target nuclei touch with each other [13 17], the strong Coulomb repulsion in the superheavy region makes a touching configuration undergo a re-separation process with a huge probability. This process is referred to as quasi-fission [18 28], and has been recognized as a primary cause of fusion inhibition in heavy systems [20, 24, 29 32]. Since quasi-fission characteristics often overlap with fission of the compound nucleus (that is, fusion-fission), formation of superheavy elements is usually identified by measuring evaporation residues of the compound nucleus, formation of which is extremely rare in the superheavy region. This makes it extremely challenging to model the formation process of superheavy nuclei and make reliable theoretical predictions for evaporation residue cross sections.

Qualitatively, the significance of quasi-fission in the different mass regions can be understood in terms of the relative position between the touching configuration and the saddle of the fission barrier. In medium-heavy systems, the saddle appears well outside the touching configuration in deformation space, and thus the compound nucleus is formed with a negligibly small probability of quasi-fission. On the other hand, in the superheavy region, the strong Coulomb repulsion leads to a lower fission barrier at a smaller deformation as compared to a fission potential in the medium-heavy region (see e.g., Fig. 7 in Ref. [33]). The touching configuration appears outside the saddle configuration, and thus a compound nucleus is formed only after the fission barrier is overcome whereas most of events lead to quasi-fission.

Based on this idea, as well as on the time-scale of each process, the formation process of evaporation residues can be conceptually divided into a sequence of the following three processes. The first is a process in which two separate nuclei form a touching configuration after overcoming the Coulomb barrier. Here, the channel coupling effects, that is, couplings of the relative motion to several nuclear collective excitations in colliding nuclei as well as several transfer processes, play an important role [13 17]. After two nuclei touch with each other, a huge number of nuclear intrinsic motions are activated and the relative energy is quickly dissipated to internal energies, landing on the right hand side of the fission barrier for a mono-nuclear system. The second stage for the formation of evaporation residues is then a diffusion over this inner barrier to form a compound nucleus, with a severe competition with the quasi-fission process. The 
third process is a statistical decay of the compound nucleus, with strong competitions between evaporation and fission.

In order to describe such a complicated process, Swiatecki et al. have proposed a simple one-dimensional model, that is, the fusion-by-diffusion model [33 35]. In this model, classical fusion cross sections with a Gaussian barrier distribution are employed for the first stage, while the second stage is modeled as a diffusion of a one dimensional parabolic barrier. Despite that the model is simple, it accounts for experimental cross sections reasonably well for $\mathrm{Pb}$ - and $\mathrm{Bi}$ - based cold fusion reactions by introducing one adjustable parameter, that is, the injection point for the second stage, or equivalently the height of the fission barrier relative to the touching configuration [33 36].

Subsequently, the fusion-by-diffusion model has been applied also to ${ }^{48} \mathrm{Ca}$-based hot fusion reactions [37, 38]. One of the characteristic features of the ${ }^{48} \mathrm{Ca}$-based hot fusion reactions [39] is that the corresponding target nuclei are in the actinide region, in which nuclei have a large deformation in the ground state. In the fusion-bydiffusion model, the effect of target deformation has been taken into account only through the Gaussian width for the barrier distribution for the first stage, even though the deformation effect may have been implicitly taken into account by a phenomenological adjustment of the injection point.

In this paper, I extend the fusion-by-diffusion model by taking into account the deformation effect both in the first and the second stages of the evaporation residue formation process. To this end, I introduce the orientation dependence to the injection point. Notice that hot fusion reactions have been, or will be, employed in order to synthesize elements beyond $\mathrm{Og}$, that is, the elements 119 and 120 [40]. The extension discussed in this paper will increase the reliability of the fusion-by-diffusion model and will provide a good guidance for future experiments. It will also help in understanding the reaction dynamics of fusion reactions of a deformed nucleus to synthesize superheavy elements. See also Refs. 41 43 for earlier publications which discussed the role of orientation of a deformed target in synthesis of superheavy elements based on a different theoretical model, that is, the dinuclear system model.

The paper is organized as follows. In Sec. II, I summarize the conventional fusion-by-diffusion model of Swiatecki et al. in order to clarify the modifications introduced in this paper. In Sec. III, I introduce the extended version of the fusion-by-diffusion model, which takes into account the deformation of a target nucleus. In Sec. IV, I apply the extended version of the model to the ${ }^{48} \mathrm{Ca}+{ }^{248} \mathrm{Cm}$ system and discuss the role of orientation of the deformed ${ }^{248} \mathrm{Cm}$ nucleus. In Sec. V, I discuss the energy dependence of the injection point, and show that the deformation effect leads to a relatively strong energy dependence. I then summarize the paper in Sec. VI.

\section{FUSION-BY-DIFFUSION MODEL}

Before I discuss the extensions of the fusion-bydiffusion model, I here summarize the current version of the model. To this end, I closely follow Ref. 35, in which the angular momentum dependence of the diffusion and the survival probabilities has been introduced to the original version of the model [33, 34]. In this $l$ dependent version of the fusion-by-diffusion model, evaporation residue cross sections $\sigma_{\mathrm{ER}}$ are evaluated as,

$$
\sigma_{\mathrm{ER}}(E)=\frac{\pi}{k^{2}} \sum_{l}(2 l+1) T_{l}(E) P_{\mathrm{fus}}(E, l) P_{\mathrm{sur}}\left(E^{*}, l\right),
$$

where $E$ is the incident energy in the center of mass frame and $k=\sqrt{2 \mu E / \hbar^{2}}$ is the corresponding wave number with $\mu$ being the reduced mass in the entrance channel. $T_{l}(E), P_{\text {fus }}(E, l)$, and $P_{\text {sur }}\left(E^{*}, l\right)$ are the probabilities for the first, the second, and the third stages, respectively, where $E^{*}$ is the excitation energy of the compound nucleus. These are the capture probability, that is, the penetrability of the Coulomb barrier, the diffusion probability of the inner fission barrier, and the survival probability of the compound nucleus against fission, respectively. I summarize each probability in the following subsections.

\section{A. Capture probability}

In the original version of the fusion-by-diffusion model, capture cross sections,

$$
\sigma_{\text {cap }}(E)=\frac{\pi}{k^{2}} \sum_{l}(2 l+1) T_{l}(E)
$$

are computed as $33,34,44]$

$$
\sigma_{\text {cap }}(E)=\int_{-\infty}^{\infty} d B f\left(B ; B_{0}\right) \sigma_{\mathrm{cl}}(E ; B),
$$

where

$$
f\left(B ; B_{0}\right)=\frac{1}{\sqrt{2 \pi} w} \exp \left[-\frac{\left(B-B_{0}\right)^{2}}{2 w^{2}}\right]
$$

represents the weight factor for a barrier distribution 45] around a mean barrier height $B_{0}$, while

$$
\sigma_{\mathrm{cl}}(E ; B)=\pi R_{b}^{2}\left(1-\frac{B}{E}\right) \theta(E-B)
$$

is the classical fusion cross section for the barrier height $B$ and the barrier position $R_{b}$. Here, $\theta(E-B)$ is the step function. With the Gaussian function for $f\left(B ; B_{0}\right)$, the integral in Eq. (3) can be evaluated analytically as 33, 34, 44]

$$
\sigma_{\text {cap }}(E)=\pi R_{b}^{2} \frac{w}{\sqrt{2 \pi} E}\left[\sqrt{\pi} x(1+\operatorname{erf}(x))+e^{-x^{2}}\right],
$$


with $x \equiv\left(E-B_{0}\right) /(\sqrt{2} w)$, where

$$
\operatorname{erf}(x)=\frac{2}{\sqrt{\pi}} \int_{0}^{x} e^{-t^{2}} d t
$$

is the error function.

In the $l$-dependent version of the fusion-by-diffusion model, the capture probability $T_{l}(E)$ is taken to be the classical one, that is, $T_{l}(E)=1$ for $l \leq l_{\max }$ and 0 for $l>l_{\max }$. The maximum angular momentum, $l_{\max }$, is determined so that the capture cross section so obtained,

$$
\sigma_{\text {cap }}(E)=\frac{\pi}{k^{2}} \sum_{l=0}^{l_{\max }}(2 l+1)=\frac{\pi}{k^{2}}\left(l_{\max }+1\right)^{2},
$$

coincides approximately with Eq. (6) for given $R_{b}$ and $B_{0}[35]$.

\section{B. Diffusion probability}

After two nuclei touch with each other by overcoming the Coulomb barrier, there is an additional inner barrier, which has to be overcome in order to form a superheavy element. In the fusion-by-diffusion model, this process is described as a diffusion of an inverted parabolic potential barrier,

$$
V_{l}(s)=V_{\text {fiss }}(s)+\frac{l(l+1) \hbar^{2}}{2 \mathcal{J}(s)} \sim V_{0 l}-C_{l}\left(s-s_{\mathrm{sd}}\right)^{2},
$$

where $s$ is the coordinate for diffusion, that is, the surface separation between the two spheres [35], $V_{\text {fiss }}(s)$ is the inner (fission) barrier, and $\mathcal{J}(s)$ is the moment of inertia for the mono-nuclear system. The last term in Eq. (9) is due to the parabolic approximation to the potential barrier around the saddle point configuration, $s_{\mathrm{sd}}$.

For a diffusion from an initial configuration $s_{\text {inj }}$ at rest, the barrier passing probability at temperature $T$ is given by 46]

$$
P_{\text {fus }}(E, l)=\frac{1}{2}\left[1-\operatorname{erf}\left(\frac{\Delta V_{l}}{T}\right)\right],
$$

in the overdamped limit, where $\Delta V_{l}$ is the effective barrier height for the second process given by $\Delta V_{l}=$ $V_{l}\left(s_{\mathrm{sd}}\right)-V_{l}\left(s_{\mathrm{inj}}\right)$ (in the fusion-by-diffusion model, the $l$-dependence in each of $s_{\mathrm{sd}}$ and $s_{\text {inj }}$ is neglected). Notice that the probability is independent of the friction coefficient and the mass parameter in the overdamped limit [46].

For given $s$ and angular momentum $l$, the temperature $T$ is estimated as

$$
T(l, s)=\sqrt{\frac{E^{*}-V_{l}(s)-E_{\mathrm{pair}}}{a(s)}},
$$

where $E_{\text {pair }}$ is the pairing energy and $a(s)$ is the level density parameter. The excitation energy $E^{*}$ is given by
$E^{*}=E-M_{\mathrm{CN}} c^{2}+M_{P} c^{2}+M_{T} c^{2}$, where $M_{\mathrm{CN}}, M_{P}$, and $M_{T}$ are the masses of the compound nucleus, the projectile nucleus, and the target nucleus, respectively. Following Ref. [35], the pairing energy, $E_{\text {pair }}$, is taken to be $21 / \sqrt{A} \mathrm{MeV}$ for even-even nucleus, where $A$ is the mass number, $10.5 / \sqrt{A} \mathrm{MeV}$ for odd mass nuclei, and 0 for odd-odd nuclei. Following again Ref. [35], I take the geometrical mean between the temperature at the saddle configuration and that at the injection point, that is, $T=\sqrt{T\left(l, s_{\mathrm{sd}}\right) T\left(l, s_{\mathrm{inj}}\right)}$, for the temperature used in Eq. (10).

For completeness, I summarize the parameterization of the inner barrier, $V_{\text {fiss }}(s)$, the moment of inertia, $\mathcal{J}(s)$, and the level density parameter, $a(s)$, in the Appendix.

\section{Survival probability}

In the superheavy region, a compound nucleus formed in a heavy-ion fusion reaction decays primarily by fission. In the fusion-by-diffusion model, the survival probability against fission is calculated using a simplified statistical model. Assuming that fission competes only with neutron emissions, the survival probability for the $N$-neutron emission channel is estimated as [35, 36, 47],

$$
\begin{aligned}
P_{\mathrm{sur}}\left(E^{*}, l\right)= & \prod_{k=1}^{N-1}\left(\frac{\Gamma_{n}^{(k)}\left(E_{k}^{*}\right)}{\Gamma_{n}^{(k)}\left(E_{k}^{*}\right)+\Gamma_{f}^{(k)}\left(E_{k}^{*}\right)}\left(1-P_{<}^{(k)}\left(E_{k}^{*}\right)\right)\right) \\
& \times \frac{\Gamma_{n}^{(N)}\left(E_{N}^{*}\right)}{\Gamma_{n}^{(N)}\left(E_{N}^{*}\right)+\Gamma_{f}^{(N)}\left(E_{N}^{*}\right)} P_{<}^{(N)}\left(E_{N}^{*}\right),
\end{aligned}
$$

where $\Gamma_{n}^{(k)}$ and $\Gamma_{f}^{(k)}$ are the neutron and the fission widths at an excitation energy $E_{k}^{*}$ after emission of $(k-1)$ neutrons, and $1-P_{<}^{(k)}$ is the probability to find the residual nucleus at excitation energies above the threshold of the next chance fission or neutron evaporation. Notice that $\Gamma_{n}^{(k)}, \Gamma_{f}^{(k)}$, and $P_{<}^{(k)}$ depend on the angular momentum $l$, but it is not expressed explicitly in Eq. (12) for simplicity of the notation.

The fission width, $\Gamma_{f}^{(k)}$, of a parent nucleus with the mass number $A_{k}$ at the excitation energy $E_{k}^{*}$ is evaluated with the transition state theory as [35],

$$
\Gamma^{(k)}\left(E_{k}^{*}\right)=\frac{1}{2 \pi \rho_{A_{k}}\left(E_{k}^{*}, s_{\mathrm{gs}}\right)} \int_{0}^{K_{\max }} d K \rho_{A_{k}}\left(K_{\max }-K, s_{\mathrm{sd}}\right),
$$

where $\rho_{A_{k}}\left(E^{*}, s_{\mathrm{gs}}\right)$ and $\rho_{A_{k}}\left(E^{*}, s_{\mathrm{sd}}\right)$ are the level densities of the parent nucleus at excitation energy $E^{*}$ at the ground state and the saddle point configurations, respectively. The maximum value of the kinetic energy for the fission degree of freedom, $K_{\max }$, is defined as,

$$
K_{\max }=E_{k}^{*}-B_{f}\left(A_{k}\right)-E_{\mathrm{rot}}\left(A_{k}, s_{\mathrm{sd}}\right)-E_{\mathrm{pair}}\left(A_{k}\right),
$$

where $B_{f}\left(A_{k}\right), E_{\text {rot }}\left(A_{k}, s_{\mathrm{sd}}\right)$, and $E_{\text {pair }}\left(A_{k}\right)$ are the fission barrier height, the rotational energy at the saddle 
point, and the pairing energy of the parent nucleus, respectively (see Sec. A-2 in the Appendix for the rotational energy). Notice that $V_{\text {fiss }}\left(s_{\text {gs }}\right)$ has to be set to zero in evaluating the level density for the ground state, as the inner barrier, $V_{\text {fiss }}(s)$, is defined with respect to the ground state energy for each nucleus. [35],

The neutron width, on the other hand, is computed as

$\Gamma_{n}^{(k)}\left(E_{k}^{*}\right)=\frac{2 m_{n} \sigma_{n}}{\pi^{2} \hbar^{2} \rho_{A_{k}}\left(E_{k}^{*}\right)} \int_{0}^{\epsilon_{\max }} \rho_{A_{k}-1}\left(\epsilon_{\max }-\epsilon_{n}\right) \epsilon_{n} d \epsilon_{n}$,

where $m_{n}$ is the neutron mass, $\sigma_{n}=\pi r_{0}^{2} A_{k}^{2 / 3}$ with $r_{0}$ $=1.45 \mathrm{fm}$ is the cross section for neutron capture, and the level densities are given by Eq. A29 with $s=s_{\text {gs }}$ for the ground state. The factor 2 in the numerator is due to the neutron spin degeneracy. The maximum neutron energy, $\epsilon_{\max }$, is given as

$\epsilon_{\max }=E_{k}^{*}-B_{n}\left(A_{k}\right)-E_{\mathrm{rot}}\left(A_{k}-1, s_{\mathrm{gs}}\right)-E_{\mathrm{pair}}\left(A_{k}-1\right)$,

where $B_{n}\left(A_{k}\right)$ is the one neutron separation energy of the parent nucleus, and $E_{\text {rot }}\left(A_{k}-1, s_{\text {gs }}\right)$ and $E_{\text {pair }}\left(A_{k}-1\right)$ are the ground state rotational energy and the pairing energy for the daughter nucleus, respectively.

A similar formula as Eq. (15) can be used to estimate the mean neutron energy for neutron emission. That is,

$$
\left\langle\epsilon_{n}\right\rangle=\frac{\int_{0}^{\epsilon_{\max }} \rho_{A_{k}-1}\left(\epsilon_{\max }-\epsilon_{n}\right) \epsilon_{n}^{2} d \epsilon_{n}}{\int_{0}^{\epsilon_{\max }} \rho_{A_{k}-1}\left(\epsilon_{\max }-\epsilon_{n}\right) \epsilon_{n} d \epsilon_{n}}
$$

This energy is used to estimate the average excitation energy of the daughter nucleus as [36],

$$
E_{k+1}^{*}=E_{k}^{*}-B_{n}\left(A_{k}\right)-\left\langle\epsilon_{n}\right\rangle
$$

with $E_{1}^{*}=E^{*}$.

The probability $P_{c}^{(k)}\left(E_{k}^{*}\right)$ in Eq. (12) is also estimated in a similar way as [35],

$$
P_{<}^{(k)}\left(E_{k}^{*}\right)=\frac{\int_{\epsilon_{\mathrm{thr}}}^{\epsilon_{\max }} \rho_{A_{k}-1}\left(\epsilon_{\max }-\epsilon_{n}\right) \epsilon_{n} d \epsilon_{n}}{\int_{0}^{\epsilon_{\max }} \rho_{A_{k}-1}\left(\epsilon_{\max }-\epsilon_{n}\right) \epsilon_{n} d \epsilon_{n}},
$$

where $\epsilon_{\mathrm{thr}}$ is the threshold energy for the next chance fission or neutron emission. It is defined as

$$
\epsilon_{\mathrm{thr}}=\epsilon_{\max }-\min \left[E_{\mathrm{thr}}^{*}(f), E_{\mathrm{thr}}^{*}(n)\right]
$$

where the function min is defined as $\min [A, B]=A$ for $A \leq B$ and $\min [A, B]=B$ for $A>B$. The threshold energy for the next chance fission, $E_{\mathrm{thr}}^{*}(f)$, is defined as,

$$
E_{\mathrm{thr}}^{*}(f)=B_{f}\left(A_{k}-1\right)+E_{\mathrm{rot}}\left(A_{k}-1, s_{\mathrm{sd}}\right)-E_{\mathrm{rot}}\left(A_{k}, s_{\mathrm{sd}}\right),
$$

where $B_{f}\left(A_{k}-1\right)$ is the fission barrier height for the daughter nucleus, and $E_{\text {rot }}\left(A_{k}-1, s_{\mathrm{sd}}\right)$ and $E_{\text {rot }}\left(A_{k}, s_{\mathrm{sd}}\right)$ are the rotational energy at the saddle point for the daughter and the parent nuclei, respectively. The threshold energy for the next chance neutron emission, $E_{\mathrm{thr}}^{*}(n)$, on the other hand, is defined as,

$E_{\mathrm{thr}}^{*}(n)=B_{n}\left(A_{k}-1\right)+E_{\mathrm{rot}}\left(A_{k}-2, s_{\mathrm{gs}}\right)-E_{\mathrm{rot}}\left(A_{k}-1, s_{\mathrm{gs}}\right)$.

$\epsilon_{\mathrm{thr}}$ is set to be zero when the value defined by Eq. (20) is negative.

\section{EXTENSION TO DEFORMED SYSTEMS}

I now discuss the extension of the fusion-by-diffusion model to deformed systems. In the original version of the model discussed in the previous section, the effect of deformation is taken into account only through the Gaussian width, w, in Eq. (44) as [33, 35],

$$
w \propto \sqrt{\frac{R_{P}^{2} \beta_{2 P}^{2}}{4 \pi}+\frac{R_{T}^{2} \beta_{2 T}^{2}}{4 \pi}+w_{0}^{2}},
$$

where $w_{0}$ is a constant and $\beta_{2 P}$ and $\beta_{2 T}$ are the quadrupole deformation parameters of the projectile and the target, respectively. The deformation effect may also be included implicitly when the injection point, $s_{\mathrm{inj}}$, is adjusted phenomenologically.

In this paper, I introduce the deformation effect more explicitly to the model. To this end, I write the evaporation residue cross sections as [15],

$$
\sigma_{\mathrm{ER}}(E)=\int_{0}^{1} d(\cos \theta) \sigma_{\mathrm{ER}}(E ; \theta)
$$

where $\theta$ is the orientation angle of a deformed target with respect to the beam direction, and $\sigma_{\mathrm{ER}}(E ; \theta)$ is the evaporation residue cross section for a fixed value of $\theta$ given by,

$\sigma_{\mathrm{ER}}(E ; \theta)=\frac{\pi}{k^{2}} \sum_{l}(2 l+1) T_{l}(E, \theta) P_{\mathrm{fus}}(E, l, \theta) P_{\mathrm{sur}}\left(E^{*}, l\right)$.

This formula is based on the isocentrifugal approximation to the angular momentum coupling [15] and on an assumption that the moment of inertia for the rotational motion is so large (therefore the energy of the first $2^{+}$ state is so small) that the orientation angle of the deformed target nucleus is fixed during fusion [15], which is well fulfilled in the actinide region. Notice that the survival probability, $P_{\text {sur }}$, remains the same as in the original version of the model, since it is related to properties of the compound nucleus, for which the memory of the entrance channel is assumed to be lost. On the other hand, the deformation effect modifies the capture probability, $T_{l}$, as well as the diffusion probably, $P_{\text {fus }}$. I will discuss below how the orientation effect can be taken into account in these probabilities. 


\section{A. Capture probability}

In order to take into account the deformation effect on the capture probability, $T_{l}$, I introduce a deformed nuclear potential of the Woods-Saxon type for the relative motion between the target and the projectile nuclei,

$$
V_{N}(r, \theta)=-\frac{V_{0}}{1+\exp \left[\left(r-R_{0}-R_{T} \sum_{\lambda} \beta_{\lambda T} Y_{\lambda 0}(\theta)\right) / a\right]},
$$

where $V_{0}, R_{0}$, and $a$ are the depth, the radius, and the diffuseness parameters, respectively, and $\beta_{\lambda T}$ are the deformation parameters of the target nucleus. The Coulomb part of the potential is also deformed as [15, 48],

$$
\begin{aligned}
V_{C}(r, \theta)= & \frac{Z_{P} Z_{T} e^{2}}{r} \\
& +\frac{3 Z_{P} Z_{T} e^{2}}{5} \frac{R_{T}^{2}}{r^{3}}\left(\beta_{2 T}+\frac{2}{7} \sqrt{\frac{5}{\pi}} \beta_{2 T}^{2}\right) Y_{20}(\theta) \\
& +\frac{3 Z_{P} Z_{T} e^{2}}{9} \frac{R_{T}^{4}}{r^{5}}\left(\beta_{4 T}+\frac{9}{7 \sqrt{\pi}} \beta_{2 T}^{2}\right) Y_{40}(\theta) \\
& +\frac{3 Z_{P} Z_{T} e^{2}}{13} \frac{R_{T}^{6}}{r^{7}} \beta_{6 T} Y_{60}(\theta),
\end{aligned}
$$

to the second order in the quadrupole deformation parameter, $\beta_{2 T}$, and the first order in the hexadecapole and the hexacontatetrapole deformation parameters, $\beta_{4 T}$ and $\beta_{6 T}$, respectively. The total potential for angular momentum $l$ reads,

$$
V(r, \theta)=V_{N}(r, \theta)+V_{C}(r, \theta)+\frac{l(l+1) \hbar^{2}}{2 \mu r^{2}},
$$

where the last term is the centrifugal potential.

I use the parabolic approximation to the potential, $V(r, \theta)$, that is, I expand the potential as

$$
V(r, \theta) \sim V_{b}(l, \theta)-\frac{1}{2} \mu \Omega(l, \theta)^{2}\left(r-R_{b}(l, \theta)\right)^{2}
$$

around the position of the Coulomb barrier, $R_{b}(l, \theta)$, for a fixed value of $\theta$. The penetrability of this potential is then computed as [15],

$$
T_{l}(E, \theta)=\frac{1}{1+\exp \left[\frac{2 \pi}{\hbar \Omega(l, \theta)}\left(V_{b}(l, \theta)-E\right)\right]} .
$$

\section{B. Diffusion probability}

The deformation effect implies that one would have to consider a diffusion in a multi-dimensional inner barrier, $V_{\text {fiss }}$, with deformation and orientation degrees of freedom, for the second stage of the evaporation residue formation process. Even though this is certainly an interesting future work, I prefer to retain here the simplicity of the fusion-by-diffusion model and thus use a (a) $\theta=0$ (tip collision)
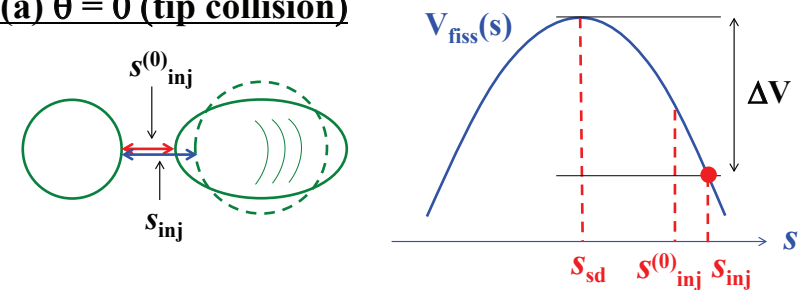

(b) $\theta=\pi / 2$ (side collision)
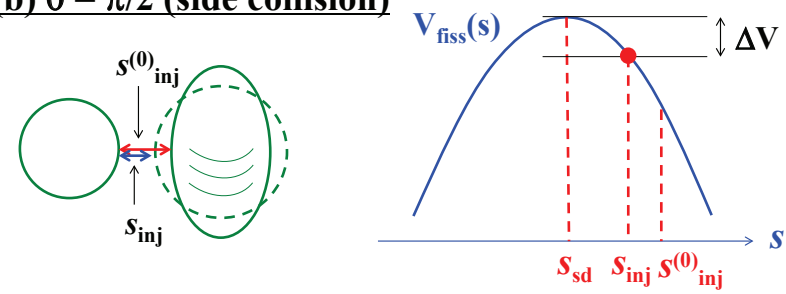

FIG. 1: A schematic illustration of the angular dependence of the injection distance, $s_{\text {inj }}(\theta)$. The upper and the lower figures show the configuration with $\theta=0$ and $\theta=\pi / 2$, respectively, where $\theta$ is the orientation angle for a prolately deformed nucleus.

one-dimensional potential. Instead, I introduce the orientation dependence to the injection point based on the notion of compactness for quasi-fission [24, 49 53].

Suppose that the target and the projectile nuclei are separated with the distance $L$ at the injection point. For a spherical target, the separation distance is then given by,

$$
L=R_{P}+R_{T}+s_{\text {inj }}^{(0)}
$$

When the target nucleus is deformed, the radius $R_{T}$ is replaced by $R_{T}(\theta)=R_{T}\left[1+\sum_{\lambda} \beta_{\lambda T} Y_{\lambda 0}(\theta)\right]$. Substituting this expression in Eq. (31), one obtains,

$$
\begin{aligned}
L(\theta) & =R_{P}+R_{T}(\theta)+s_{\mathrm{inj}}^{(0)}, \\
& =R_{P}+R_{T}+s_{\mathrm{inj}}^{(0)}+R_{T} \sum_{\lambda} \beta_{\lambda T} Y_{\lambda 0}(\theta) .
\end{aligned}
$$

This implies that the orientation dependent injection parameter is given by,

$$
s_{\mathrm{inj}}(\theta)=L(\theta)-R_{P}-R_{T}=s_{\mathrm{inj}}^{(0)}+R_{T} \sum_{\lambda} \beta_{\lambda T} Y_{\lambda 0}(\theta) .
$$

This is schematically illustrated in Fig. 1. The diffusion probability is then given by

$$
P_{\text {fus }}(E, l, \theta)=\frac{1}{2}\left[1-\operatorname{erf}\left(\frac{\Delta V_{l}(\theta)}{T(\theta)}\right)\right],
$$

where both the effective barrier height, $\Delta V_{l}$, and the temperature, $T$, depend on the angle $\theta$ through the angle dependent injection point, $s_{\text {inj }}(\theta)$. A similar idea was employed also in Ref. [54] in more realistic Langevin calculations. 


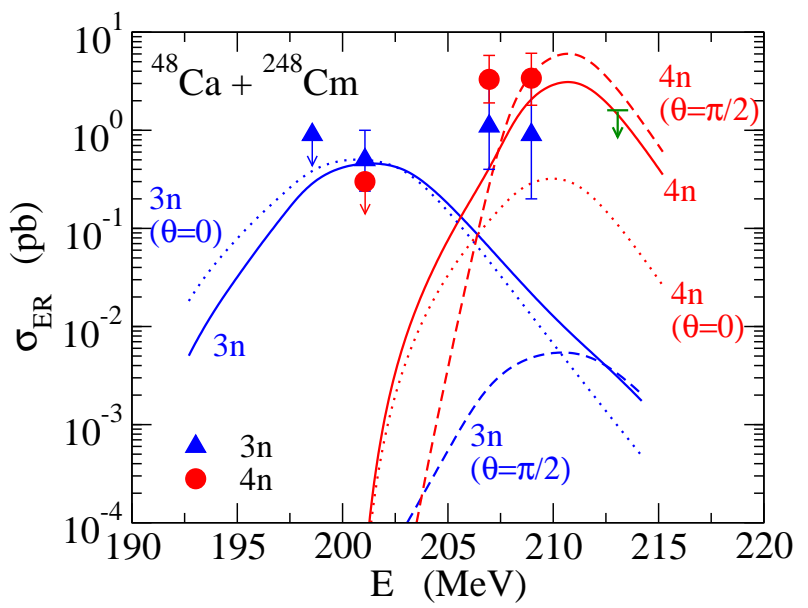

FIG. 2: The evaporation residue cross sections for the ${ }^{48} \mathrm{Ca}+{ }^{248} \mathrm{Cm}$ system as a function of the incident energy in the center of mass frame obtained with the extended fusionby-diffusion model with deformations of the target nucleus. Eq. 36 is used for the energy dependence of the injection point. The dotted and the dashed lines show the cross sections for the orientation angle of $\theta=0$ and $\theta=\pi / 2$, respectively, while the solid lines are obtained by taking an average over all the angles, $\theta$. The experimental data are taken from Refs. [62, 63].

\section{APPLICATION TO ${ }^{48} \mathrm{Ca}+{ }^{248} \mathrm{Cm}$ REACTION}

Let us now apply the extended fusion-by-diffusion model discussed in the previous section to the ${ }^{48} \mathrm{Ca}+{ }^{248} \mathrm{Cm}$ reaction, for which the barrier distribution for the capture process has recently been measured [55] using the quasi-elastic scattering [56, [57]. The theoretical analysis for the measured barrier distribution has clearly shown that the maximum of the evaporation residue cross sections for this system appears at an energy slightly above the barrier height for the side collision, in good agreement with the notion of compactness 24, 49 53. The aim of this section is to gain a deeper insight into the effect of orientation of the deformed ${ }^{248} \mathrm{Cm}$ nucleus by re-analyzing the evaporation residue cross sections using the extended fusion-by-diffusion model.

In the calculation presented below, I use the deformation parameters of $\beta_{2 T}=0.297, \beta_{4 T}=0.039$, and $\beta_{6 T}=-0.035$ together with the radius of $R_{T}=1.2 A_{T}^{1 / 3} \mathrm{fm}$ for the entrance channel. The value of $\beta_{2 T}$ is estimated from the measured electric transition probability 58], while the values of $\beta_{4 T}$ and $\beta_{6 T}$ are taken from Ref. [59]. For the Woods-Saxon potential, I use the parameters of $V_{0}=70 \mathrm{MeV}, R_{0}=1.18 \times\left(48^{1 / 3}+248^{1 / 3}\right) \mathrm{fm}$, and $a=0.69$ $\mathrm{fm}$, which is similar to the one used in Ref. [55] for the coupled-channels analysis for the quasi-elastic barrier distribution for this system, with a slight re-adjustment in order to reproduce the measured capture cross sections [60]. The deformation parameters and the shell correction energies, both at the ground state and at the saddle point, as well as the ground state masses and the fission

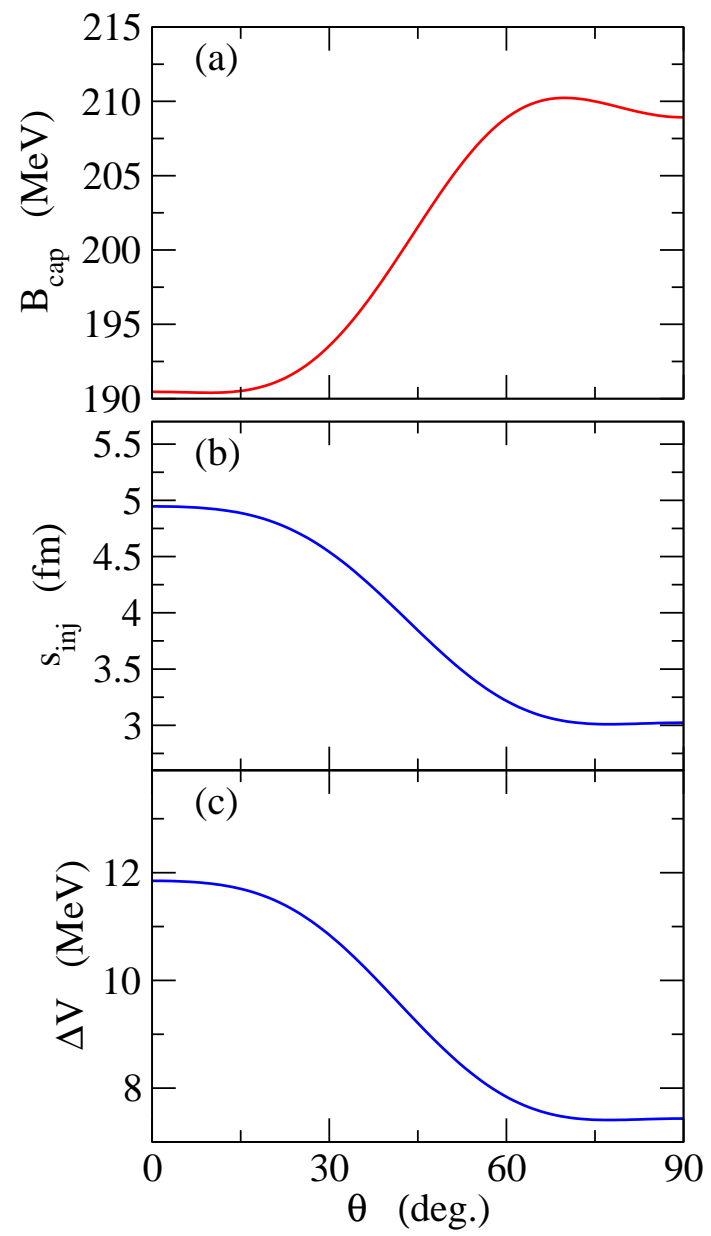

FIG. 3: The dependence of (a) the capture barrier height, (b) the injection distance, and (c) the height of the diffusion barrier on the orientation angle of the deformed target nucleus for the ${ }^{48} \mathrm{Ca}+{ }^{248} \mathrm{Cm}$ reaction. The injection distance and the height of the diffusion barrier are evaluated at energy $E=215$ $\mathrm{MeV}$ in Eq. (36).

barrier heights are all taken from Ref. 61]. This mass table lists the values only for even-even nuclei, and thus for odd-mass nuclei I take an average of the values for the neighboring nuclei. I assume that the shell correction energy is negligible at the injection point. Following Refs. [35, 37], I introduce a liner energy dependence to the injection point, $s_{\text {inj }}^{(0)}$, in Eq. (34), which is specified below.

The solid lines in Fig. 2 show the evaporation residue cross sections obtained with

$$
s_{\text {inj }}^{(0)}=4.698 \mathrm{fm}-0.16\left(E-B_{0}\right) \mathrm{fm} / \mathrm{MeV},
$$

where the reference barrier height, $B_{0}$, is given by [35]

$$
B_{0}=0.853315 z+0.0011695 z^{2}-0.000001544 z^{3} \mathrm{MeV},
$$

with $z=Z_{P} Z_{T} /\left(A_{P}^{1 / 3}+A_{T}^{1 / 3}\right)$. In order to compare with the experimental data [62, 63], I smear the calculated 


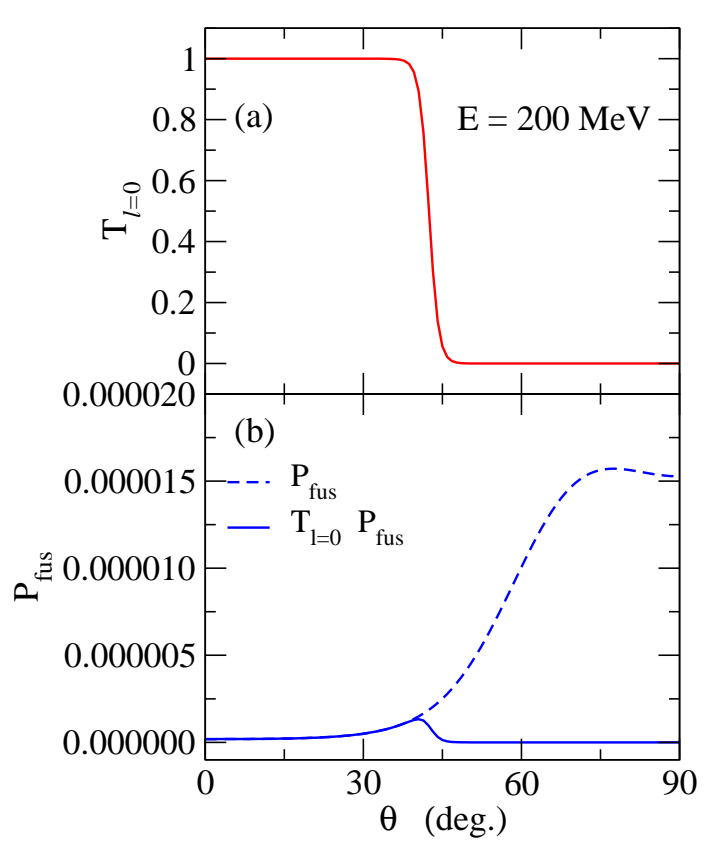

FIG. 4: The dependence of (a) the capture probability, $T_{l=0}$, and (b) the diffusion probability, $P_{\text {fus }}$, on the orientation angle of the deformed target nucleus for the $s$-wave ${ }^{48} \mathrm{Ca}+{ }^{248} \mathrm{Cm}$ reaction at $E=200 \mathrm{MeV}$. In the panel (b), the compound nucleus formation probability, defined as a product of $T_{l=0}$ and $P_{\text {fus }}$ is also shown by the solid line.

cross sections as,

$$
\bar{\sigma}_{\mathrm{ER}}(E)=\frac{1}{\Delta E} \int_{E-\Delta E / 2}^{E+\Delta E / 2} \sigma_{\mathrm{ER}}\left(E^{\prime}\right) d E^{\prime},
$$

in order to take into account a loss of the beam energy in the target material with a finite thickness [35]. According to Refs. 62, 63], I take $\Delta E=5.4$ and $3.4 \mathrm{MeV}$ for the $3 n$ and $4 n$ evaporation channels, respectively, even though different values for $\Delta E$ should be used for different experimental runs. The figure also shows the cross section for $\theta=0$ and $\theta=\pi / 2$ by the dotted and the dashed lines, respectively (see Eq. (25)). One can see that the $4 n$ channel is mainly due to the side collision with $\theta=\pi / 2$, while the $3 n$ channel is mainly due to the tip collision with $\theta=0$. The former result is consistent with the earlier experimental conclusions in Ref. [24, 49 52, 55].

The energy dependence of the relative contribution for the side and the tip collisions can be understood in terms of the angle dependence of the capture and the diffusion barriers. The top panel of Fig. 3 shows the height of the capture barrier as a function of the orientation angle. For nuclei with prolate deformation, the barrier is lower for the tip collision $(\theta=0)$ and increases with $\theta$ (the figure shows a non-monotonic behavior due to the finite value of $\beta_{6}$ deformation). Therefore, the side collision is suppressed at low energies. The middle and the bottom panels show the injection distance and the barrier height for the diffusion process, respectively, at $E=215$

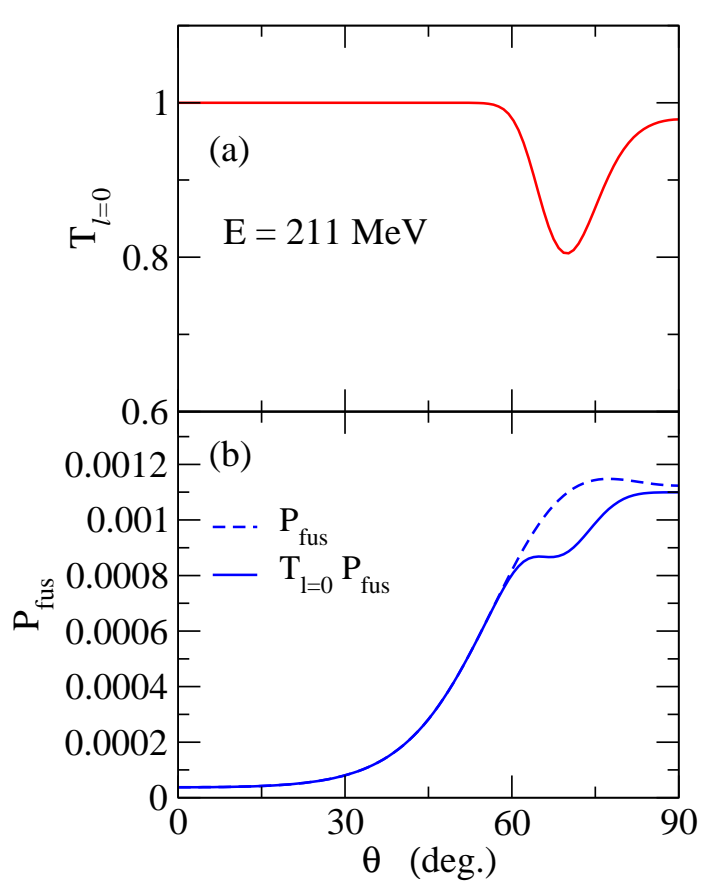

FIG. 5: Same as Fig. 4, but at $E=211 \mathrm{MeV}$.

$\mathrm{MeV}$. The injection distance is small for the side collision, and thus the diffusion barrier is low. This leads to an enhancement of the diffusion probability for the side collision as compared to that for the tip collision. The side collision then becomes dominant at high energies, where the suppression due to the capture process is small.

In order to demonstrate this more explicitly, Figs. 4 and 5 show the capture, the diffusion, and the compound nucleus formation probabilities for $l=0$ as a function of the orientation angle, $\theta$, at $E=200$ and $211 \mathrm{MeV}$, respectively. Here, the compound nucleus formation probability is defined as a product of the capture and the diffusion probabilities. For $E=200 \mathrm{MeV}$ shown in Fig. 4 , the capture barrier is higher than the incident energy for $\theta>43 \mathrm{deg}$. (see Fig. 3(a)), and the capture probability drops off abruptly in this range of orientation angle. The contribution of the side collision is then negligible even though the diffusion probability itself is relatively large, as shown in the lower panel of Fig. 4. On the other hand, for $E=211 \mathrm{MeV}$ shown in Fig. 5, the capture probability is close to unity except for the angles around $\theta \sim 70$ deg. (again, the non-monotonic behavior is due to the finite value of $\beta_{6}$ ), and the side collision competes well with the tip collision in the capture stage of the reaction. The side collision then gives the largest contribution to the compound nucleus formation, since the diffusion probability is large due to a small injection distance. A qualitatively similar conclusion has been obtained also with the dinuclear system model [43]. 


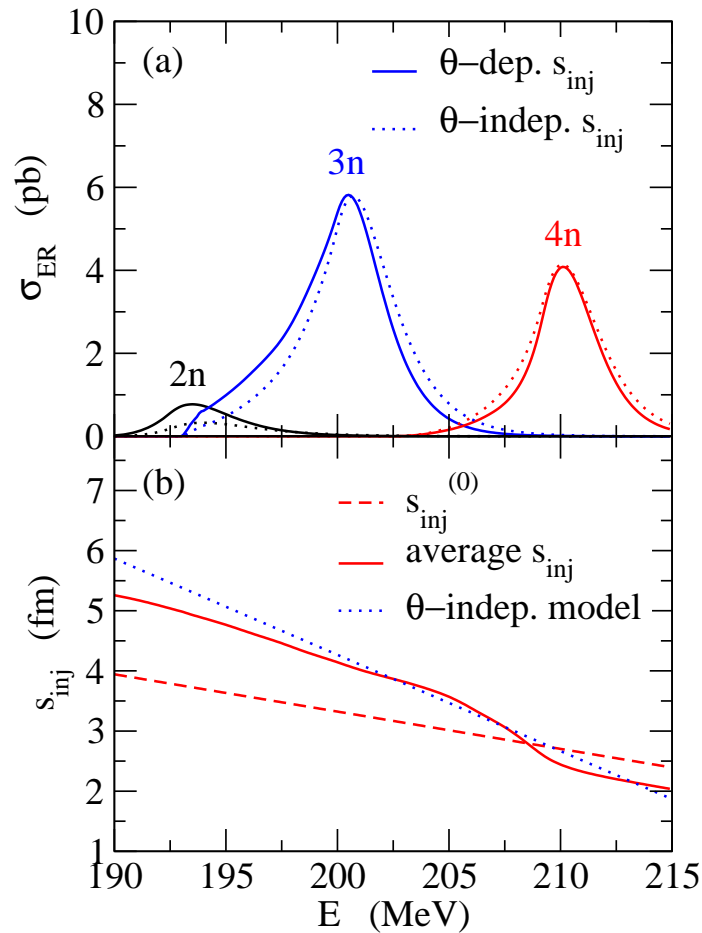

FIG. 6: (The upper panel) The evaporation residue cross sections for the ${ }^{48} \mathrm{Ca}+{ }^{248} \mathrm{Cm}$ system obtained with the extended version of the fusion-by-diffusion model with Eq. (40) (the solid lines), in comparison with the results obtained by neglecting the angle dependence of the injection distance (the dotted lines). (The lower panel) The energy dependence of the injection distance. The dashed line shows the energy dependence given by Eq. (40), while the solid line takes into account the deformation effect with Eq. (42). The dotted line shows the energy dependence for the angle independent model, that corresponds to the dotted lines in the upper panel.

\section{ENERGY DEPENDENCE OF THE INJECTION DISTANCE}

The evaporation residue cross sections for the ${ }^{48} \mathrm{Ca}+{ }^{248} \mathrm{Cm}$ system obtained with the original version of fusion-by-diffusion model are shown in Fig. 2 (d) in Ref. 37]. In order to draw this figure, the authors of Ref. [37] used the parameterization of the injection distance given by,

$$
s_{\text {inj }}=4.09 \mathrm{fm}-0.192\left(E-B_{0}\right) \mathrm{fm} / \mathrm{MeV} .
$$

Notice that this energy dependence of the injection distance is much stronger than the one used for cold fusion reactions, that is, $s_{\mathrm{inj}}=2.30 \mathrm{fm}-0.062\left(E-B_{0}\right) \mathrm{fm} / \mathrm{MeV}$ [35].

A similar quality of the result to the one obtained with the original version of the model can be obtained with the extended version of the model discussed in this paper using

$$
s_{\text {inj }}^{(0)}=3.457 \mathrm{fm}-0.062\left(E-B_{0}\right) \mathrm{fm} / \mathrm{MeV},
$$

as shown by the solid lines in the upper panel of Fig. 6. Notice that this has the same energy dependence of $s_{\text {inj }}$ as the one for cold fusion reactions discussed in Ref. [35]. If the angle dependence of $s_{\mathrm{inj}}(\theta)$ is disregarded in the present model, similar results are obtained with a stronger dependence, that is,

$$
s_{\text {inj }}(\theta)=4.613 \mathrm{fm}-0.16\left(E-B_{0}\right) \mathrm{fm} / \mathrm{MeV},
$$

as shown by the dotted lines in the figure. Therefore, the energy dependence of the injection distance is indeed weakened if the deformation effect is explicitly taken into account.

In order to discuss this point more clearly, I take an average of $s_{\text {inj }}(\theta)$ with the total evaporation residue cross sections for each angle $\theta, \sigma_{\mathrm{ER}}(\theta)$, as a weight factor. That is, I define the average injection distance as,

$$
\bar{s}_{\mathrm{inj}}(E) \equiv \frac{\int_{0}^{1} d(\cos \theta) s_{\mathrm{inj}}(\theta) \sigma_{\mathrm{ER}}(E ; \theta)}{\int_{0}^{1} d(\cos \theta) \sigma_{\mathrm{ER}}(E ; \theta)} .
$$

This quantity is shown by the solid line in the lower panel of Fig. 6. For a comparison, the figure also shows the energy dependences given by Eqs. (40) and (41) with the dashed and the dotted lines, respectively. One can clearly see from the figure that the angle dependence of the injection distance provides a strong energy dependence of an effective injection distance, which is compatible with the energy dependence obtained with the angle-independent model shown by the dotted line. Evidently, the strong energy dependence found in Ref. [37] for hot fusion reactions mocks up the deformation effect of the target nuclei to a large extent, which are not included explicitly in the original version of the fusion-by-diffusion model.

\section{SUMMARY}

By taking into account the effects of deformation of the target nucleus, I have extended the fusion-by-diffusion model of Swiatecki et al. for heavy-ion fusion reactions to synthesize superheavy elements. To this end, I have introduced the angle dependence to the injection distance, based on the notion of compactness for quasi-fission. I have also used the barrier distribution for the capture process which is consistent with the rotational coupling of a deformed nucleus. I have applied the extended version of the fusion-by-diffusion model to the hot fusion reaction ${ }^{48} \mathrm{Ca}+{ }^{248} \mathrm{Cm}$ and found that the maximum of evaporation residue cross sections appears at an energy slightly above the Coulomb barrier for the side collision. At this energy, the capture probability is close to unity, while the diffusion probability is large for the side collision due to a compactness of the touching configuration. At lower energies, the side collision is largely suppressed because of a high capture barrier, and the tip collision gives an important contribution.

I have also discussed the energy dependence of the injection distance. I have argued that a strong energy de- 
pendence shows up when the deformation effect is converted to an effective energy dependence. This observation is consistent with the strong energy dependence found in the previous analyses for hot fusion reactions with the original version of the fusion-by-diffusion model.

In this paper, following the philosophy of the fusion-bydiffusion model, I considered a diffusion of a simple one dimensional inner barrier, for which the deformation effect is taken into account only through the injection point for diffusion. In reality, however, it is not obvious at all how the diffusion path is evolved in a multi-dimensional energy surface with deformation and orientation degrees of freedom. In particular, as the nuclear deformation is a quantal effect, it is expected that the deformation will be reduced or even disappears during the heat-up process after the contact of two colliding nuclei. It would remain a theoretical challenge to model the shape evolution of the dinuclear system towards a compound nucleus by taking into account the gradual change of the deformation in a hot target-like nucleus. To address this question, one would need to develop a quantum theory of friction, such as the ones discussed in Ref. [64]. Obviously, much more work is necessary towards this goal and to gain a deep insight into the reaction dynamics of heavy-ion fusion reactions for the synthesis of superheavy nuclei.

\section{Acknowledgments}

I thank T. Tanaka, T. Ichikawa, Y. Abe, and Y. Aritomo for useful discussion. I thank also D.J. Hinde for his useful comments and careful reading of the manuscript. This work was supported by JSPS KAKENHI Grant Number 17K05455.

\section{Appendix A: Inputs to the fusion-by-diffusion model}

For completeness, in this Appendix, I summarize the inputs to the fusion-by-diffusion model in order to clarify the notations. Even though these can be found in Refs. [33 35], it is useful to summarize them here in a selfcontained manner, since a different version of the model may have used a different parameterization.

\section{Inner barrier for diffusion}

Consider the situation where the projectile and the target nuclei, whose radius is $R_{P}$ and $R_{T}$, respectively, are separated with a distance $L$ with some appropriate neck in between them 65. In terms of the separation distance, $s \equiv L-R_{P}-R_{T}$, between the two fragments, the inner barrier for $s>0$ for the diffusion process is parametrized as,

$$
V_{\text {fiss }}(s)=E_{\text {surf }}\left[a+b \frac{s}{R}+c\left(\frac{s}{R}\right)^{2}\right],
$$

where $E_{\text {surf }}$ and $R$ are the surface energy and the radius of the compound nucleus, respectively. The saddle of this potential appears at $s_{\mathrm{sd}}=-R b /(2 c)$.

With the atomic number $Z\left(=Z_{P}+Z_{T}\right)$, the neutron number $N\left(=N_{P}+N_{T}\right)$, and the mass number $A\left(=A_{P}+\right.$ $\left.A_{T}\right)$ for the compound nucleus, $E_{\text {surf }}$ and $R$ are taken to be 33,34 ]

$$
\begin{aligned}
R & =1.155 A^{1 / 3} \quad(\mathrm{fm}), \\
E_{\text {surf }} & =17.9439\left(1-1.7826 I^{2}\right) A^{2 / 3}
\end{aligned}
$$

with $I=(N-Z) / A$. The constant $a$ in Eq. A1D are taken to be $a=\alpha_{1}+\alpha_{2}(1-x)+\alpha_{3}(1-x)^{2}$ with [35],

$$
\begin{aligned}
& \alpha_{1}=-0.00564-0.01936 e^{-D / 0.02240}, \\
& \alpha_{2}=0.05122+0.11931 e^{-D / 0.03800}, \\
& \alpha_{3}=-0.07424+0.95959 D
\end{aligned}
$$

where $x$ is the fissility parameter of the compound nucleus and $D$ is the asymmetry parameter defined as

$$
D=\left(\frac{R_{P}-R_{T}}{R_{P}+R_{T}}\right)^{2}
$$

The radii are computed as $R_{i}=1.155 A_{i}^{1 / 3}(\mathrm{fm})$ with the mass number for each fragment, $A_{i}(i=P, T)$, and the fissility parameter is given as [33, 34],

$$
x=\frac{Z^{2} / A}{50.883\left(1-1.7826 I^{2}\right)} .
$$

The other parameters $b$ and $c$ in Eq. (A1) are given in a similar way as

$$
b=\beta_{1}+\beta_{2}(1-x)+\beta_{3}(1-x)^{2},
$$

with

$$
\begin{aligned}
& \beta_{1}=-0.06080+1.137825 D-10.7077 D^{2} \\
& \beta_{2}=0.27691-2.93119 D+12.60944 D^{2} \\
& \beta_{3}=-0.02398-1.14854 D
\end{aligned}
$$

and

$$
c=\gamma_{1}+\gamma_{2}(1-x)+\gamma_{3}(1-x)^{2},
$$

with

$$
\begin{aligned}
& \gamma_{1}=-0.02722+0.2231 D, \\
& \gamma_{2}=0.02050+0.32122 D, \\
& \gamma_{3}=0.03843+1.03731 D .
\end{aligned}
$$

For $s<0$, the potential is taken to be 35]

$$
\begin{aligned}
V_{\text {fiss }}(s)= & E_{\text {surf }}\left[\left(\frac{b}{S_{0}}+\frac{3 a}{S_{0}^{2}}\right)\left(\frac{s}{R}-S_{0}\right)^{2}\right. \\
& \left.+\left(\frac{b}{S_{0}^{2}}+\frac{2 a}{S_{0}^{3}}\right)\left(\frac{s}{R}-S_{0}\right)^{3}\right],
\end{aligned}
$$

with the same coefficients $a, b$, and $c$ as in Eq. A1 and

$$
S_{0} \equiv \frac{2 R-2\left(R_{P}+R_{T}\right)}{R} .
$$




\section{Moment of inertia}

The moment of inertia $\mathcal{J}(s)$ is necessary in order to evaluate the rotational energy,

$$
E_{\mathrm{rot}}(s)=\frac{l(l+1) \hbar^{2}}{2 \mathcal{J}(s)}
$$

in Eq. (9). In the fusion-by-diffusion model, the rigidbody moment of inertia is employed. For the injection point, the moment of inertia reads 35.,

$$
\mathcal{J}\left(s_{\text {inj }}\right)=\mu r^{2}+\frac{2}{5} M_{P} R_{P}^{2}+\frac{2}{5} M_{T} R_{T}^{2},
$$

where $\mu=M_{P} M_{T} /\left(M_{P}+M_{T}\right)$ is the reduced mass and $r=R_{P}+R_{T}+s_{\text {inj }}$ is the distance between the projectile and the target nuclei at the injection point. On the other hand, for the saddle configuration, the moment of inertia is evaluated as,

$\mathcal{J}\left(s_{\mathrm{sd}}\right)=\frac{1}{5} M_{\mathrm{CN}} R^{2}\left[\left(1+\alpha_{\mathrm{sd}}\right)^{2}+\left(1+\alpha_{\mathrm{sd}}\right)^{-1}\right]+2 M_{\mathrm{CN}} b_{f}^{2}$,

where the constant $b_{f}$ is taken to be $1 \mathrm{fm}$ [35]. In this equation, $\alpha_{\mathrm{sd}}$ is the deformation at the saddle point given as,

$$
\alpha_{\mathrm{sd}}=\sum_{\lambda} \beta_{\lambda}^{(\mathrm{sd})} Y_{\lambda 0}(\theta=0)=\sum_{\lambda} \sqrt{\frac{2 \lambda+1}{4 \pi}} \beta_{\lambda}^{(\mathrm{sd})},
$$

where the deformation parameters, $\beta_{\lambda}^{(\mathrm{sd})}$, can be extracted from a mass model. The moment of inertia for the ground state configuration is also evaluated in a similar way as in Eq. A21 using the ground state deformation, $\alpha_{\mathrm{gs}}$, instead of $\alpha_{\mathrm{sd}}$.

Notice that $\alpha_{\text {sd }}$ given by Eq. A22 can also be used to estimate the value of $s_{\mathrm{sd}}$. For a deformed configuration at the saddle point, the length of the nucleus along the longer axis reads,

$2 R_{\max }=2 R\left(1+\sum_{\lambda} \beta_{\lambda}^{(\mathrm{sd})} Y_{\lambda 0}(\theta=0)\right)=2 R\left(1+\alpha_{\mathrm{sd}}\right)$.
Assuming that this configuration is realized with the projectile and the target nuclei separated with the separation distance $s_{\mathrm{sd}}$ leads to the following relation between $s_{\mathrm{sd}}$ and $\alpha_{\mathrm{sd}}$,

$$
s_{\mathrm{sd}}=2 R_{\max }-2 R_{P}-2 R_{T}=R S_{0}+2 R \alpha_{\mathrm{sd}},
$$

where $S_{0}$ is given by Eq. A18. This $s_{\mathrm{sd}}$ is used when the saddle point of the potential, $V_{\text {fiss }}(s)$, defined in the previous subsection is smaller than $S_{0}$, which is usually the case in the superheavy region.

\section{Level density parameter}

Disregarding the shell effects, the level density parameter $\tilde{a}(s)$ at $s$ is taken as [35, 66],

$$
\tilde{a}(s)=a_{V} A+a_{S} A^{2 / 3} B_{S}+a_{C} A^{1 / 3} B_{K},
$$

with $a_{V}=0.0696 \mathrm{MeV}^{-1}, a_{S}=0.1801 \mathrm{MeV}^{-1}$, and $a_{C}=0.1644 \mathrm{MeV}^{-1}$. The surface function, $B_{S}$, and the curvature function, $B_{K}$, are given by

$$
\begin{aligned}
& B_{S}=1+\left(0.6416 \alpha-0.1421 \alpha^{2}\right)^{2}, \\
& B_{K}=1+\left(0.6542 \alpha-0.0483 \alpha^{2}\right)^{2}
\end{aligned}
$$

with $\alpha=\left(s / R-S_{0}\right) / 2($ see Eq. (A24) $)$.

The shell effect on the level density parameter can be taken into account using the prescription of Ignatyuk et al. 67, that is,

$$
a(s)=\tilde{a}(s)\left[1+\frac{E_{\text {shell }}(s)}{U(s)}\left(1-e^{-U(s) / E_{D}}\right)\right]
$$

where $U(s)=E^{*}-V_{l}(s)-E_{\text {pair }}$ (see Eq. (9)) and the shell damping energy is taken to be $E_{D}=18.5 \mathrm{MeV}$ [35, 66]. Here, $E_{\text {shell }}(s)$ is the shell correction energy, which can be extracted from a mass model.

The level density is then given as

$$
\rho(s)=(\text { const. }) \times e^{2 \sqrt{a(s) U(s)}},
$$

whereas the nuclear temperature $T(l, s)$ is estimated using Eq. (11).
[1] S. Hofmann and G. Münzenberg, Rev. Mod. Phys. 72, 733 (2000).

[2] J.H. Hamilton, S. Hofmann, and Y.T. Oganessian, Ann. Rev. Nucl. Part. Sci. 63, 383 (2013).

[3] Special issue on superheavy nuclei, ed. by C.E. Düllmann, R.-D. Herzberg, W. Nazarewicz, and Y. Oganessian, Nucl. Phys. A944, 1 (2015).

[4] M.R. Mumpower, T. Kawano, T.M. Sprouse, N. Vassh, E.M. Holmbeck, R. Surman, and P. Möller, arXiv:1802.04398.

[5] Yu. Ts. Oganessian et al., Phys. Rev. C74, 044602
(2006).

[6] K. Morita et al., J. Phys. Soc. Jpn. 73, 2593 (2004); 76, 045001 (2007); 81, 103201 (2012).

[7] Yu. Ts. Oganessian et al. Phys. Rev. C69, 021601(R) (2004); Phys. Rev. C76, 011601(R) (2007).

[8] Yu. Ts. Oganessian et al., Phys. Rev. Lett. 104, 142502 (2010).

[9] V.I. Zagrebaev and W. Greiner, Nucl. Phys. A944, 257 (2015).

[10] V.I. Zagrebaev, Y. Aritomo, M.G. Itkis, Y.T. Oganessian, and M. Ohta, Phys. Rev. C65, 014607 (2001). 
[11] Y. Aritomo, T. Wada, M. Ohta, and Y. Abe, Phys. Rev. C59, 796 (1999).

[12] C.W. Shen, G. Kosenko, and Y. Abe, Phys. Rev. C66, 061602 (2002).

[13] A.B. Balantekin and N. Takigawa, Rev. Mod. Phys. 70, 77 (1998).

[14] M. Dasgupta, D.J. Hinde, N. Rowley, and A.M. Stefanini, Annu. Rev. Nucl. Part. Sci. 48, 401 (1998).

[15] K. Hagino and N. Takigawa, Prog. Theor. Phys. 128, 1061 (2012).

[16] B.B. Back, H. Esbensen, C.L. Jiang, and K.E. Rehm, Rev. Mod. Phys. 86, 317 (2014).

[17] G. Montagnoli and A.M. Stefanini, Eur. Phys. J. A53, 169 (2017).

[18] J. Tōke, R. Bock, G.X. Dai, A. Gobbi, S. Gralla, K.D. Hildenbrand, J. Kuzminski, W.F.J. Müller, A. Olmi, and H. Stelzer, Nucl. Phys. A440, 327 (1985).

[19] B.B. Back, Phys. Rev. C31, 2104 (1985).

[20] D.J. Hinde, R.G. Thomas, R. du Rietz, A. Diaz-Torres, M. Dasgupta, M.L. Brown, M. Evers, L.R. Gasques, R. Rafiei, and M.D. Rodriguez, Phys. Rev. Lett. 100, 202701 (2008).

[21] R. du Rietz, E. Williams, D.J. Hinde, M. Dasgupta, M. Evers, C.J. Lin, D.H. Luong, C. Simenel, and A. Wakhle, Phys. Rev. C88, 054618 (2013).

[22] E. Williams, D.J. Hinde, M. Dasgupta, R. du Rietz, I.P. Carter, M. Evers, D.H. Luong, S.D. McNeil, D.C. Rafferty, K. Ramachandran, and A. Wakhle, Phys. Rev. C88, 034611 (2013).

[23] J. Khuyagbaatar, D.J. Hinde, I.P. Carter, M. Dasgupta, Ch. E. Düllmann, M. Evers, D.H. Luong, R. du Rietz, A. Wakhle, E. Williams, and A. Yakushev, Phys. Rev. C91, 054608 (2015).

[24] D.J. Hinde, D.Y. Jeung, E. Prasad, A. Wakhle, M. Dasgupta, M. Evers, D.H. Luong, R. du Rietz, C. Simenel, E.C. Simpson, and E. Williams, Phys. Rev. C97, 024616 (2018).

[25] E.M. Kozulin, G.N. Knyazheva, K.V. Novikov, I.M. Itkis, M.G. Itkis, S.N. Dmitriev, Yu. Ts. Oganessian, A.A. Bogachev, N.I. Kozulina, I. Harca, W.H. Trzaska, and T.K. Ghosh, Phys. Rev. C94, 054613 (2016).

[26] G.N. Knyazheva, E.M. Kozulin, R.N. Sagaidak, A. Yu. Chizhov, M.G. Itkis, N.A. Kondratiev, V.M. Voskressensky, A.M. Stefanini,B.R. Behera, L. Corradi, E. Fioretto, A. Gadea, A. Latina, S. Szilner, M. Trotta, S. Beghini, G. Montagnoli, F. Scarlassara, F. Haas, N. Rowley, P.R.S. Gomes, and A. Szanto de Toledo, Phys. Rev. C75, 064602 (2007).

[27] K. Nishio, S. Mitsuoka, I. Nishinaka, H. Makii, Y. Wakabayashi, H. Ikezoe, K. Hirose, T. Ohtsuki, Y. Aritomo, and S. Hofmann, Phys. Rev. C86, 034608 (2012).

[28] A.N. Andreyev, K. Nishio, and K.-H. Schmidt, Rep. Prog. in Phys. 81, 016301 (2018).

[29] C.-C. Sahm, H.-G. Clerc, K.-H. Schmidt, W. Reisdorf, P. Armbruster, F.P. Hessberger, J.G. Keller, G. Münzenberg, and D. Vermeulen, Nucl. Phys. A441, 316 (1985).

[30] D.J. Hinde, M. Dasgupta, and A. Mukherjee, Phys. Rev. Lett. 89, 282701 (2002).

[31] A.C. Berriman, D.J. Hinde, M. Dasgupta, C.R. Morton, R.D. Butt, and J.O. Newton, Nature 413, 144 (2001).

[32] R. Yanez, W. Loveland, J.S. Barrett, L. Yao, B.B. Back, S. Zhu, and T.L. Khoo, Phys. Rev. C88, 014606 (2013).

[33] W.J. Swiatecki, K. Siwek-Wilczynska, and J. Wilczynski,
Phys. Rev. C71, 014602 (2005).

[34] W.J. Swiatecki, K. Siwek-Wilczynska, and J. Wilczynski, Acta Phys. Pol. B 34, 2049 (2003).

[35] T. Cap, K. Siwek-Wilczynska, and J. Wilczynski, Phys. Rev. C83, 054602 (2011).

[36] T. Ichikawa and A. Iwamoto, J. Phys. Soc. Jpn. 79, 074201 (2010).

[37] K. Siwek-Wilczynska, T. Cap, M. Kowal, A. Sobiczewski, and J. Wilczynski, Phys. Rev. C86, 014611 (2012).

[38] T. Cap, K. Siwek-Wilczynska, M. Kowal, and J. Wilczynski, Phys. Rev. C88, 037603 (2013).

[39] Yu. Ts. Oganessian and V.K. Utyonkov, Nucl. Phys. A944, 62 (2015).

[40] C.E. Düllmann, EPJ Web of Conf. 163, 00015 (2017).

[41] A. Nasirov, A. Fukushima, Y. Toyoshima, Y. Aritomo, A. Muminov, S. Kaladarov, and R. Utamuratov, Nucl. Phys. A759, 342 (2005).

[42] N. Wang, J.-q. Li, and E.-g. Zhao, Phys. Rev. C78, 054607 (2008).

[43] L. Zhu, Z.-Q. Feng, C. Li, and F.-S. Zhang, Phys. Rev. C90, 014612 (2014).

[44] K. Siwek-Wilczynska and J. Wilczynski, Phys. Rev. C69, 024611 (2004).

[45] N. Rowley, G.R. Satchler, and P.H. Stelson, Phys. Lett. B254, 25 (1991).

[46] Y. Abe, D. Boilley, B.G. Giraud, and T. Wada, Phys. Rev. E61, 1125 (2000).

[47] T. Cap, K. Siwek-Wilczynska, I. Skwira-Chalot, and J. Wilczynski, Acta Phys. Pol. B 43, 297 (2012).

[48] K. Hagino, N. Rowley, and A.T. Kruppa, Comp. Phys. Comm. 123, 143 (1999).

[49] D.J. Hinde, M. Dasgupta, J.R. Leigh, J.P. Lestone, J.C. Mein, C.R. Morton, J.O. Newton, and H. Timmers, Phys. Rev. Lett. 74, 1295 (1995).

[50] D.J. Hinde, M. Dasgupta, J.R. Leigh, J.C. Mein, C.R. Morton, J.O. Newton, and H. Timmers, Phys. Rev. C53, 1290 (1996).

[51] K. Nishio, H. Ikezoe, S. Mitsuoka, I. Nishinaka, Y. Nagame, Y. Watanabe, T. Ohtsuki, K. Hirose, and S. Hofmann, Phys. Rev. C77, 064607 (2008).

[52] K. Nishio, H. Ikezoe, S. Mitsuoka, and J. Lu, Phys. Rev. C62, 014602 (2000).

[53] N. Rowley, N. Grar, and M. Trotta, Phys. Rev. C76, 044612 (2007).

[54] Y. Aritomo, K. Hagino, K. Nishio, and S. Chiba, Phys. Rev. C85, 044614 (2012).

[55] T. Tanaka, Y. Narikiyo, K. Morita, K. Fujita, D. Kaji, K. Morimoto, S. Yamaki, Y. Wakabayashi, K. Tanaka, M. Takeyama, A. Yoneda, H. Haba, Y. Komori, S. Yanou, B.J.-P. Gall, Z. Asfari, H. Faure, H. Hasebe, M. Huang, J. Kanaya, M. Murakami, A. Yoshida, T. Yamaguchi, F. Tokanai, T. Yoshida, S. Yamamoto, Y. Yamano, K. Watanabe, S. Ishizawa, M. Asai, R. Aono, S. Goto, K. Katori, and K. Hagino, J. Phys. Soc. Jpn. 87, 014201 (2018).

[56] H. Timmers, J.R. Leigh, M. Dasgupta, D.J. Hinde, R.C. Lemmon, J.C. Mein, C.R. Morton, J.O. Newton, and N. Rowley, Nucl. Phys. A584, 190 (1995).

[57] K. Hagino and N. Rowley, Phys. Rev. C69, 054610 (2004).

[58] S. Raman, C.W. Nestor, Jr., and P. Tikkanen, At. Data Nucl. Data Tables 78, 1 (2001).

[59] P. Möller, A.J. Sierk, T. Ichikawa, and H. Sagawa, At. Data Nucl. Data Tables 109-110, 1 (2016). 
[60] E.M. Kozulin, G.N. Knyazheva, I.M. Itkis, M.G. Itkis, A.A. Bogachev, E.V. Chernysheva, L. Krupa, F. Hanappe, O. Dorvaux, L. Stuttge, W.H. Trzaska, C. Schmitt, and G. Chubarian, Phys. Rev. C90, 054608 (2014).

[61] M. Kowal, P. Jachimowicz, and J. Skalski, arXiv:1203.5013

[62] S. Hofmann et al., Eur. Phys. J. A48, 62 (2012).

[63] Yu. Ts. Oganessian et al., Phys. Rev. C70, 064609
(2004); Phys. Rev. C63, 011301 (2000).

[64] M. Tokieda and K. Hagino, Phys. Rev. C95, 054604 (2017).

[65] D. Boilley, H.L. Lu, C.W. Shen, Y. Abe, and B.G. Giraud, Phys. Rev. C84, 054608 (2011).

[66] W. Reisdorf, Z. Phys. A300, 227 (1981).

[67] A.V. Ignatyuk, G.N. Smirenkin, and A.S. Tishin, Yad. Fiz. 21, 485 (1975); Sov. J. Nucl. Phys. 21, 255 (1975). 